Murillo-Zamora, C. (2021). Gestión de fronteras marítimas: el caso de Costa Rica y Nicaragua en el océano Pacífico. Revista de Biología Tropical, 69(Suppl. 2), S1-S14. https:// doi.org/10.15517/rbt.v69iS2.48125

Biologín Tropical

https://doi.org/10.15517/rbt.v69iS2.48125

\title{
Gestión de fronteras marítimas: el caso de Costa Rica y Nicaragua en el océano Pacífico
}

\author{
Carlos Murillo-Zamora ${ }^{1,2,3}$; (iD https://orcid.org/0000-0001-5104-7675 \\ 1. Observatorio del Desarrollo, Universidad de Costa Rica, San José, Costa Rica; carlos.murillozamora@ucr.ac.cr, \\ camuza@gmail.com \\ 2. Escuela de Administración Pública, Universidad de Costa Rica, San José, Costa Rica. \\ 3. Escuela de Relaciones Internacionales, Universidad Nacional, Heredia, Costa Rica.
}

Recibido 15-XII-2020. Corregido 29-I-2021. Aceptado 16-II-2021.

\begin{abstract}
Management of maritime boundaries: the case of Costa Rica and Nicaragua in the Pacific

Introduction: Public management has acquired new characteristics because of change in the way governance is approached. As the subject areas, levels and scales of this management have expanded, public administration has become more complex, requiring new approaches. Although the Law of the Sea and the thesis that the land dominates the sea are not new, the truth is that it was with the United Nations Convention of the Law of the Sea, of 1982, that the areas under the jurisdiction of the coastal States were formalized and institutionalized. This has generated new challenges for the governments, since not only does territorial sovereignty extend over a large portion of the sea, but new responsibilities are assumed on behalf of the international community.

Objective: The matter links public management with the regulations of the Law of the Sea regarding the delimitation and administration of boundaries. Therefore, this review proposes an approach to a matter that has not been explored enough.

Methods: A review of the literature on public border management was made, considering the case of maritime boundaries, identifying international jurisprudence -especially of the International Court of Justice- and actions implemented by Costa Rica.

Results: in the land portion, border management shows important development; this is not the case in the maritime spaces. The jurisdiction is different in each of these spaces, so public management must take these particularities into account. Things get complicated when it comes to the border area, because in it the marine and the maritime converge, but also different interests, due to the nature of the marine space. Therefore, it is necessary to recognize that the management of maritime boundaries is not as simple as moving what is done on land to the sea. When the limit was defined by a bilateral treaty, some provisions facilitate cross-border coordination and cooperation. For this reason, the case of Costa Rica and Nicaragua in the Pacific Ocean was used as a reference, since in this case the dividing line was established by the International Court of Justice, which causes a layout that is not necessarily satisfactory for the actors involved.

Conclusions: the differences in border management between land and sea, in addition to how the boundary between the two countries is defined, has made it difficult for Costa Rica to implement public management, which is the best-known experience in the region on marine-maritime policies and management.
\end{abstract}

Key words: Law of the Sea; maritime boundaries; public management; Costa Rica; Nicaragua; Pacific Ocean. 
Desde la perspectiva de la Administración Pública y la gestión de fronteras la atención sobre el espacio geográfico ha estado centrada en la porción terrestre del territorio del Estado; mientras que el límite, en términos de definición del espacio físico que sirve de escenario a tal gestión, establece los puntos de interacción con terceros, sean otros Estados o la comunidad internacional. Así una línea limítrofe define hasta dónde llega la gestión pública y la acción gubernamental -ejercicio del gobierno y la gobernación-; por ende, el ámbito en donde tiene lugar la dimensión interna de la soberanía. Esto a pesar de que desde la Antigüedad persiste la tesis de que la tierra domina el mar; sin embargo, este -en aquellos sectores bajo jurisdicción del Estado ribereño- no es incorporado como ámbito de acción de lo público. Esto ni en el sentido de administrar y gestionar (realización de las decisiones directivas de los gobiernos) ni de la esencia de la gobernanza, según se define más adelante.

Con la suscripción, en 1982, de la Convención de Naciones Unidas para el Derecho del Mar se reconoce, formalmente en un texto de carácter constitucional, la jurisdicción del Estado ribereño sobre las aguas interiores, mar territorial, zona económica exclusiva y plataforma continental. Esto extendió la gestión pública más allá de la porción terrestre del territorio, incorporando espacios que requieren de una administración y una gobernanza particular, pues se ejerce sobre un escenario con una dimensión dual: marina y marítima.

Considerando que esa cuestión no ha recibido la atención por parte de la mayoría de los gobiernos, y que desde la perspectiva académica implica que no se ha profundizado en el ámbito de la gestión pública marina-marítima, sobre todo en lo relativo a las fronteras de los espacios marítimos, en este trabajo primero reviso lo relativo a gestión pública. En una segunda sección hago referencia a la cuestión del territorio y las fronteras, complementado -en un tercera sección- con las fronteras marítimas, seguido de lo relativo a delimitación -una materia clave para extender los procesos que asumen los Estados ribereños para establecer los espacios en donde ejerce derechos diversos. Ello conduce, en una cuarta sección la gobernanza marítima y marina. En la última sección abordo el tema de las fronteras Costa Rica y Nicaragua en el océano Pacífico.

Como se trata de una cuestión que vincula la gestión pública con la normativa del derecho del mar en materia de delimitación y administración de fronteras; este trabajo busca establecer, a partir de la revisión de literatura, una relación entre la gestión pública de fronteras terrestres y la situación de fronteras en los espacios marítimos, que resulta un tema poco explorado. Se complementa esa revisión con la consideración de jurisprudencia internacional sobre delimitación marítima, particularmente el caso entre Costa Rica y Nicaragua en el océano Pacífico.

\section{MATERIALES Y MÉTODOS}

Se recurre a la revisión de literatura sobre gestión pública, como punto de partida para entender la administración de las fronteras terrestres, sobre lo cual existe alguna experiencia en el ámbito de la Administración Pública. Sobre esa base se establece un vínculo con la gestión de fronteras marítimas, un tema prácticamente dejado de lado por los especialistas en la administración del espacio territorial estatal. A partir de ese contexto se alude a la cuestión de la gobernanza, sobre todo en lo marítimo. Con ese marco referencial se analiza la sentencia de la Corte Internacional de Justicia sobre el diferendo limítrofe entre Costa Rica y Nicaragua, específicamente en el océano Pacífico.

\section{RESULTADOS Y DISCUSIÓN}

\section{Gestión pública}

La gestión pública ha dejado de ser una cuestión limitada a la acción de los asuntos propios de los entes gubernamentales y de la burocracia; es decir, no se restringe a lo que esta realice, sino que contempla dirección estratégica y de la calidad del proceso, por lo cual tiene que ver con los esquemas de gestión 
del desempeño y del conocimiento (Aguilar, 2016). En ese contexto surge el denominado "momento posdecisional", relacionado con la comunicación/ argumentación, la implementación y la evaluación de las políticas (Aguilar, 2016).

Por consiguiente, hoy la gestión pública hace referencia a toda actividad del Estado dirigida a lograr objetivos o intereses sociales, requiriendo una apropiada dirección y ejecución de las decisiones políticas a través de una estructura institucional organizada bajo un sistema jurídico normativo preestablecido $\mathrm{y}$ dotado de recursos humanos y financieros (Organización Internacional para las Migraciones [OIM], 2012). Por ende, tiene una dimensión operativa y funcional, relacionada con la administración pública. De ahí que la gestión pública sea el medio para la implementación y administración de políticas públicas, por lo que sin buenas políticas no puede haber buena gestión. También es un asunto de gestión de la calidad, porque la complejidad obliga a los gobiernos a buscar herramientas y mecanismos para lograr sus objetivos y realizar las tareas de forma eficaz, dando lugar a lo que se denomina la nueva gestión pública (Moyado, 2011). Ello implica cambios profundos en la administración de los recursos estatales.

Como la gestión pública tiene que ver con los aspectos técnicos de la gobernanza, basta con aludir al modo posgubernamental denominado nueva gobernanza, que redimensiona la administración de lo público y la capacidad de acción del gobierno (Aguilar, 2010a, p. 189). Este nuevo modo de cómo se gobierna una sociedad y se resuelven sus problemas resulta de la acción colectiva en red, en el marco de governance system (Aguilar, 2010a).

Para una buena gestión pública, sobre todo en términos de la "nueva gestión pública", es necesario tener claro el escenario en el que se implementan las políticas públicas, el entorno y el contexto; así como los actores participantes y sus intereses e interacciones con otros agentes. La gestión no actúa en el vacío, ni es un esquema estrictamente top-down. Esto es significativo en la gestión de los espacios marinos y marítimos, sobre todo en zonas fronterizas. Entiendo un espacio marítimo como el área geográfica sobre la cual un Estado ribereño ejerce algún tipo de jurisdicción y tiene obligaciones sobre el recurso marino (Langlet, 2018).

Lo anterior hace necesario señalar que no se trata solo de la gestión pública, sino de la acción gubernamental, pues el gobierno gobierna el territorio, la sociedad y las relaciones exteriores a través de las entidades administrativas, civiles, policiales y militares; esto significa que tal acción tiene implicaciones administrativas, pues es el aparato de la administración pública quien ejecuta políticas, programas, proyectos y otras iniciativas para lograr los fines u objetivos que contribuyen al desarrollo integral del país. Y lo anterior se relaciona con la gobernanza, el determinante de la nueva gestión pública, vinculado con la administración pública. Por ende, la cuestión de los asuntos transfronterizos y en general territoriales demanda una atención particular, y esto se enmarca en lo que se denomina new public governance, que está relacionada con el modo o proceso de gobernar asociado, colaborativo, concertado y corresponsable (ver la sección sobre gobernanza marina y marítima).

\section{Territorio y fronteras: un asunto de construcción de los espacios}

El mundo está en una época de transformación del Estado y sus dinámicas, sobre todo por los procesos de desnacionalización y globalización; sin embargo, permite la estrecha relación entre territorio, autoridad y derechos, con una compleja interacción entre lo local/ nacional y lo global, haciendo más complejos los procesos asentados en la visión estatocéntrica del "Estado territorial" (Sassen, 2013). Pero, al mismo tiempo, el territorio es espacio social para la materialización de relaciones de poder y de interacciones entre agentes (Spíndola, 2016); por lo que es central a la complejidad de las interacciones sociales, económicas, políticas, culturales y estratégicas, no es solo espacio físico (Vargas, 2012). Por eso no hay que considerar solo el territorio, sino también la 
territorialidad y de ahí a la cuestión de la frontera, como espacio de separación/vinculación. Mientras que el Estado aparece como “....aparato institucional políticamente construido para administrar un territorio delimitado por la frontera como realidad jurídica" (Spíndola, 2016).

El territorio no se limita a la dimensión geográfica, sino que se expresa en las dimensiones económica, social, ambiental y organizativa, siendo apropiado por los actores para generar construcciones colectivas, de forma que resulta en "...un espacio pluridimensional que se construye a partir de una base económico-productiva determinada y de las estrategias desplegadas por actores" (Martínez, 2012).

Así surgen nuevas geografías, con elementos territoriales y electrónicos (producto de una imbricación entre lo digital y lo no digital) que traspasan fronteras y órdenes espacio-temporales, generando ensamblajes complejos en los que se insertan redes transfronterizas, y aparecen -la "proximidad desterritorializada"- espacios estratégicos subnacionales, vinculados por flujos intensos de operaciones de espacialidad y temporalidad desnacionalizados, que no son del todo nacionales ni del todo globales (Sassen, 2013).

En ese escenario se producen las relaciones entre lo marino y lo marítimo; la gestión pública de fronteras y de la planificación espacial marina; la relación entre el territorio y la territorialidad nacional soberana; las acciones de las comunidades locales y aquellas internacionales/globales; y las actividades de sectores específicos. Así en el espacio territorial tienen lugar numerosas dinámicas que tienen que ver con la gobernabilidad y la gobernanza; y, por ende, en el proceso de toma de decisiones y formulación de políticas públicas.

Desde esa perspectiva hay que tener en cuenta las interacciones en la porción emergida del territorio estatal, como también en las aguas jurisdiccionales. De forma que el Estado tiene varias dimensiones interconectadas: terrestre, marina, costera y marino-costera. Ello está vinculado con acciones concretas como el establecimiento de áreas naturales protegidas, investigación científica, actividades turísticas, protección de recursos naturales (vivos y no vivos), infraestructuras y rutas marítimas, entre muchas otras.

Al ser el territorio un espacio construido, con distintas dinámicas, es necesaria la planificación estratégica territorial. Esta se constituye en un recurso de gestión pública para identificar las características particulares de los diferentes espacios territoriales y su territorialidad, sobre todo aquellos comunitarios, a ser considerados en los niveles de la división político-administrativa. Tal planificación territorial debe estar directamente conectada con los planes nacionales y las estrategias de desarrollo (cfr. OIM, 2012).

En tal planificación territorial interviene el rol de la cultura, nacional y local, y la cosmovisión de la relación entre comunidad y medio natural social (el ser humano en su entorno natural), que se relaciona con la teoría de las aglomeraciones (concentraciones socioeconómicas y polos de poder) (Elizalde, 2003). Por lo que esa planificación se convierte en un instrumento de la gobernanza local (Flores, 2014), vinculando las políticas públicas, la gestión pública y la gobernanza multinivel.

No se trata de pensar en el territorio como si fuera un espacio aislado, pues es una categoría que enlaza diferentes dimensiones sobre la base de relaciones (Flores, 2014). El territorio está conectado al de otros Estados. Por eso es necesario considerar los contextos de frontera, en donde tienen lugar vínculos jurídicos, lazos históricos, económicos y sociales, movilidad humana transfronteriza; pero también otros tipos de flujos considerados ilícitos. Entonces, la frontera corresponde a aquella porción del territorio vinculada al límite entre dos o más unidades territoriales estatales o entre una unidad territorial y la comunidad internacional, en donde tienen lugar procesos humanos con efectos económicos, sociales, culturales, políticos, jurídicos y ambientales (OIM, 2012). Por lo que no se puede obviar el "modelo de territorio", que resulta de una descripción del futuro que esperan las personas y comunidades en el marco de un entendimiento mutuo 
de lo económico, social, cultural y ambiental (Flores, 2014).

Mientras que el limite hace referencia a la línea divisoria imaginaria que separa dos unidades territoriales estatales soberanas y la frontera constituye una porción de territorio vinculada los límites en donde se desarrollan procesos humanos en contextos geográficos e históricos determinados (OIM, 2012). Ahora bien, la línea limítrofe es un asunto de competencia del gobierno central; mientras que en las áreas fronterizas en la porción terrestre las competencias son compartidas entre lo nacional y lo local/regional (Oddone, 2014).

A lo anterior se suma el concepto de fronteras vivas, constituidas por espacios con dinámicas económicas y sociales permanentes y cotidianas. Esto contrasta con aquellas fronteras estáticas de zonas despobladas y sin mayor vinculación económica, política o estratégica. Se suman las fronteras abiertas, en donde tienen lugar relaciones entre poblaciones locales y las acciones en los distintos niveles de la administración pública (Oddone, 2014).

La delimitación territorial no es estática, más bien es la base para la construcción de los procesos de desarrollo y tiene que ver lo espacial geográfico, la acción político-administrativa y la interacción entre actores y escalas (Flores, 2014). Por eso las fronteras están vinculadas con la formación del Estado nacional, con la fragmentación territorial, con conflictos armados y por supuesto con la construcción de la identidad nacional (Oddone, 2014). Buen ejemplo es el golfo de Fonseca (El Salvador, Honduras y Nicaragua), tanto en la parte terrestre como marítima.

El trazado de una frontera tiene repercusiones económicas, sociales, ambientales, culturales y políticas. Por consiguiente, cuando se habla hoy de territorio de fronteras es necesario pensar en la cooperación transfronteriza, en lo macro y micro regional, así como en los procesos de reterritorialización y de integración regional y territorial, al mismo tiempo que de descentralización de la gestión pública (Caleta et al., 2013).
Un tema que es necesario puntualizar, antes de abordar el tema de las fronteras marítimas, es la diferencia entre lo marino y lo marítimo del territorio. El espacio marino corresponde al escenario natural independiente de la acción humana, por lo que se requiere pensar en la unidad de los océanos y su hábitat, y carece de fronteras político-administrativas. Mientras que el espacio marítimo es aquel generado por decisiones políticas, económicas, sociales, jurídicas y estratégicas, basado en la tesis del derecho del mar de que la tierra domina el mar (Murillo, 2017). Los distintos ámbitos bajo jurisdicción nacional y fuera de esta establecidos por la Convención de Naciones Unidas sobre el Derecho del Mar (Convemar) de 1982, son el resultado de siglos de interés de los Estados ribereños por ejercer algún tipo de control sobre los mares y océanos. Y como en donde hay una actividad humana es necesario establecer normas jurídicas para regularla, bajo el principio ubi societas, ibi jus, resulta más relevante en los océanos, porque estos “... han sido el escenario o el camino de grandes empresas humanas, exploración, encuentro de culturas, conquista, colonización e inevitablemente, también de choques y conflictos" (Lacleta, 2003).

\section{Fronteras marítimas: su definición y gestión}

Los mares son clave para la seguridad de los Estados, pero también para su desarrollo y prosperidad, pues los espacios marinos son fuente de riqueza económica, bienestar social y cultural; pero también el Estado ribereño tiene obligaciones en términos medioambientales y marítimos (por las actividades lícitas e ilícitas que se realicen en esas zonas bajo jurisdicción nacional), por eso no se debe considerar solo la gestión del espacio marítimo, sino el control de las fronteras, como una manifestación de la soberanía estatal, pero también una obligación con la comunidad internacional (Sobrino \& Oanta, 2010). Según Convemar en algunas actividades en los espacios marinos bajo 
jurisdicción nacional el Estado actúa como representante de la comunidad internacional.

La frontera marítima es "...la línea de separación entre zonas marítimas de soberanía y/o jurisdicción de dos o más Estados vecinos o no pertenecientes a ningún Estado $\mathrm{y}$, por tanto, zonas internacionales" (Sobrino \& Oanta, 2010). Estas divisiones son significativas, producto del problema político y jurídico del reparto de los mares (Lacleta, 2003), por lo que el fundamento de la gestión de los espacios marítimos y sus fronteras radica en Convemar $\mathrm{y}$ otros convenios que norman las distintas actividades en los territorios marítimos bajo jurisdicción del Estado ribereño y más allá (me refiero a la altamar y su suelo y subsuelo, denominado en el derecho del mar como "La Zona", patrimonio común de la humanidad).

La frontera marítima ha evolucionado en las últimas décadas, lo que hace necesario diferenciar entre aquellas restrictivas y las que tienen una dinámica amplia. Esto porque hay diferencias, en términos jurisdiccionales entre los diferentes espacios: aguas interiores, mar territorial, zona contigua, zona económica exclusiva y plataforma continental, en donde el Estado ribereño ejerce soberanía, derechos de soberanía y jurisdicción. Pero más allá de esto la característica es que tal frontera carece de una definición precisa y porosa (Sobrino \& Oanta, 2010). Sin olvidar la dificultad para ejercer el control y vigilancia por el tamaño de los espacios marítimos y la longitud del límite.

Pero en donde hay fronteras hay delimitación -entendida como la serie de acciones tendientes a establecer una línea divisoria, teniendo en cuenta la normativa vigente (Orihuela, 2017)-. En el mar es más complejo, porque no hay fronteras naturales (basadas en accidentes geográficos). Tal proceso de delimitación se torna diverso, difícil y conflictivo (Orihuela, 2017), porque no es viable trazar líneas divisorias en función de accidentes geográficos, geomorfológicos o geológicos del fondo marino (Lacleta, 2003).

Ello ha dado lugar al desarrollo del campo de la delimitación marítima, una parte especifica del derecho del mar, que atiende tres aspectos limítrofes básicos: i) entre Estados adyacentes; ii) Estados frente a frente; y iii) con la altamar. Hay una serie de principios, partiendo de la premisa de que "...la tierra domina al mar y que aquella brinda cobertura jurídica a los derechos que los ribereños pueden ejercer sobre el medio marino adyacente a su costa" (Orihuela, 2017). Por otra parte, la delimitación se basa en las tesis de equidistancia y equidad, aunque los Estados son soberanos para definir sus fronteras a partir de otros criterios. Sin embargo, se considera que en todo momento debe respetarse la tesis de non-cut off o "no cierre del frente costero" de un territorio ribereño (cfr. Acikgonul, 2016).

\section{Delimitación marítima: \\ el componente clave}

La definición del territorio, en cuanto espacio de acción, es el punto de partida para una buena gestión. Los límites y fronteras permiten al tomador de decisiones reconocer hasta dónde alcanza su acción y en dónde comienza la interacción con actores extranjeros. En el caso del mar esto es más significativo, porque se trata de espacios en que el trazado de la línea divisoria es más complejo por lo fluido del escenario. No hay que olvidar que esto implica un componente internacional, porque “...la delimitación no podría depender de la sola voluntad del Estado ribereño tal y como se llegare a expresar en su derecho interno... [por lo que] la validez de delimitación frente a Estados terceros depende incuestionablemente del derecho internacional", como lo reconoció la Corte Internacional de Justicia en el caso de las pesquerías entre Reino Unido y Noruega (citado en Gómez-Robledo, 2016). De ahí que sea una operación internacional con carácter nacional, que no puede ser efectuada unilateralmente e involucra la noción de soberanía (Haque \& Hassan, 2014a). Ello ha conducido a generar un amplio conjunto de normas sobre delimitación que han sido codificadas en los tratados (División de Asuntos Oceánicos y del Derecho del Mar [DOALOS], 2001), que se denominan los principios y reglas del derecho 
internacional público para la delimitación de áreas marítimas entre dos o más Estados (Prescott \& Schofield, 2005).

El punto de partida de la delimitación marítima es la línea de base a partir de la cual se miden los espacios bajo jurisdicción nacional; pero que también define las aguas interiores. Convemar (art. 5 y 7) reconoce las líneas de base normal o línea de bajamar a lo largo de la costa y las líneas de base recta que une puntos apropiados a lo largo de la costa. La importancia de la claridad y precisión del trazado de las líneas de base es porque determina el borde exterior de cada espacio marítimo y, por ende, el tipo de jurisdicción que el Estado ribereño ejerce. Sobre el mar territorial (12 millas marinas de ancho) se posee soberanía, que abarca el espacio aéreo y el suelo y subsuelo (art. 2). En la zona contigua (24 millas marinas) hay jurisdicción en asuntos aduaneros, fiscales, de inmigración y sanitarios (art. 33). Respecto a la ZEE (art. 56) se reconocen derechos de soberanía para la exploración y explotación, conservación y administración de recursos naturales (vivos y no vivos) y jurisdicción sobre islas artificiales, investigación científica marina y protección y preservación del medio marino. Y en el caso de la plataforma continental existen derecho de soberanía exclusivos (art. 77), que son independientes de la ocupación real o efectiva.

Al trazar los límites, ya sea entre Estados con costas adyacentes, frente a frente o respecto a la altamar se han reconocido una serie de criterios; porque esto se genera por el traslape de derechos (entitlements) de Estados ribereños soberanos (Evans, 2016). Predominan los factores geográficos costeros y a partir de estos se aplica la equidistancia y la equidad (Olorundami, 2017; DOALOS, 2001), a los que se ha sumado el concepto de proporcionalidad o falta de desproporcionalidad como criterio para evaluar la equidad (DOALOS, 2001).

Esos principios son los que determinan los criterios y métodos para trazar las fronteras, pero dependen de la existencia de islas, rocas o elevaciones en bajamar y hasta de la morfología del suelo marino; sin embargo, como señala
A. Gómez-Robledo (2016): “...la delimitación debe ser realizada por la aplicación de criterios equitativos y por la utilización de métodos prácticos aptos para asegurar un resultado equitativo, habida cuenta de la configuración geográfica de la región y de otras circunstancias pertinentes". En última instancia es una decisión política, que depende de la existencia de una voluntad política, con el objetivo de "lograr un resultado equitativo" (DOALOS, 2001). Como decisión política una opción para llegar a establecer la división es una negociación entre las partes involucradas, dos o más -hay una tendencia a favorecer procesos bilaterales-; eso garantiza un resultado aceptado por las partes. Cuando no se logra por esa vía, se recurre a instancias judiciales internacionales -tribunales y arbitrajes- o a la mediación de una tercera parte. El problema es que se pueden generar insatisfacciones si alguno de los Estados considera que fue afectado en sus intereses territoriales.

En ese sentido hay una gran diferencia respecto a la delimitación terrestre. Sin embargo, no hay que perder de vista que bajo la tesis de que la tierra domina el mar, lo que se hace es la territorialización de los mares (Cadena \& Devia, 2012).

$\mathrm{Y}$ esa complejidad se ve incrementada por el cambio climático, porque el aumento del nivel del mar tiene un impacto sobre las líneas de base. Un cambio en estas implica variaciones en el trazado de las fronteras, más crítico en el caso de Estados insulares que pudieran tener partes de su territorio sumergidas (Haque \& Hassan, 2014b; cfr. Caron, 2009). Esto hace que lo que puede denominarse "derecho de delimitación marítima" enfrenta desafíos en el siglo XXI, como también puede afectar “.... la sostenibilidad y consistencia del orden legal internacional" (Haque \& Hassan, 2014b). De esta forma hay una estrecha relación entre fronteras, gestión y gobernanza.

\section{Gobernanza marítima y marina}

Al vincular la gestión de fronteras con lo marítimo y lo marino es necesario aludir a 
la gobernanza en esos dos ámbitos; porque la gobernanza está relacionada estrechamente con el espacio y su delimitación, así como con la administración de las unidades, sean naturales (ecosistemas marinos) o legales (áreas bajo jurisdicción estatal). La gobernanza está vinculada con la gestión de redes en las que intervienen distintos actores y temáticas (Oddone, 2014); pues se entiende como “...el proceso mediante el cual los actores de una sociedad deciden sus objetivos de convivencia -fundamentales y coyunturales- y las formas de coordinarse para realizarlos: su sentido de dirección y de capacidad de dirección" (Aguilar, 2016). Como indiqué se relaciona con la gestión pública y con el proceso de gobernar y la capacidad y eficacia de los gobiernos (Aguilar, 2010b; Aguilar, 2016).

El concepto de gobernanza adquiere auge en la década de 1990 bajo la tesis de gobernar sin gobierno (Rhodes, 1996), adquiriendo nuevas dimensiones, por lo que se incorpora al análisis de los ecosistemas terrestres, reconociendo las preocupaciones por factores económicos, social y conductuales, la tecnología, el conocimiento y la toma de decisiones relacionadas para atender los principales desafíos ambientales, desarrollando lo relativo a "gobernanza ambiental" (Lemons \& Agrawal, 2006). De ahí se pasó a la cuestión de la gobernanza ambiental transfronteriza, para atender la cuestión de conflictos socioambientales por el desarrollo de actividades transfronterizas y sus efectos ambientales y políticos en los ámbitos jurisdiccionales estatales (Lizama, 2017). En este sentido se ha llegado a redefinir la gobernanza en términos de "....un proceso complejo y distribuido que fundamentalmente determina cómo los recursos son utilizados y protegidos y cómo los beneficios y servicios relacionados son distribuidos en la sociedad", lo cual adquiere más importancia en el caso de los océanos, sobre todo por las especies migratorias (Henshaw, 2018).

Por consiguiente, se trata de la interacción entre lo administrativo/legal, la gestión de recursos y la gobernanza, junto con lo ecosistémico en un espacio delimitado (Langlet, 2018).
Tal enfoque de gestión ecosistémica ha evolucionado en términos de lo que Pavlikakis y Tsihrintzis (citados Kern \& Söderström, 2018) definen como “...un enfoque holístico para la administración, que está basado en ciertos principios e incluye elementos que determina la metodología a seguir, con soluciones alcanzables, socialmente aceptadas concernientes a los problemas de desarrollo y protección de una región".

Pero la complejidad también radica en los desafíos que significa la gestión de recursos marinos y costeros, la cual se caracteriza por los niveles de incertidumbre y procesos interconectados en múltiples escalas (ecológica, jurisdiccional, social) y los niveles (local, nacional, regional y global), por lo que es necesario implementar una gestión proactiva y precautoria basada en información disponible de distintas fuentes, aun cuando no todas las causas y efectos sean conocidos (Baldwin, Mahon, \& McConney, 2013).

Ello da lugar al establecimiento de enfoques para la gestión de los espacios, tal como el enfoque de administración basada en el ecosistema. Según el Programa de Evaluación de Aguas Transfronterizas del PNUMA hay 66 grandes ecosistemas marinos, y en Centroamérica se identifican tres. Uno es del Pacífico, abarca desde México hasta Perú y el principal riesgo para los recursos hídricos es la inadecuada gobernanza, seguido de lo socioeconómico y lo biofísico (Global Water Partnership Centroamérica, 2019). Esto hace necesario pensar en términos transfronterizos y de los sistemas socio-ecológicos, que están vinculados con los marcos de gobernanza local y nacional (Baldwin et al, 2013). La cuestión es que no siempre la arquitectura institucional que gestiona los grandes ecosistemas contribuye a la buena gobernanza (Hearns, Henshaw, \& Paisley, 2014).

Se ha establecido el Principio de Malawi para el enfoque ecosistémico, planteado en el marco de la Convención sobre Biodiversidad Biológica, que tiene en cuenta los espacios y las escalas, así como las "fronteras biofísicas" (Langlet, 2018). Esto conduce a la relación 
entre el espacio natural, el ecosistema y la construcción de lo marítimo sobre el que se formulan las políticas públicas; es decir, la gobernanza marítima/marina opera en un espacio y una escala que combina elementos y mecanismos que se construyen sobre ámbitos específicos. De ahí la importancia de entender la gestión de las fronteras marítimas, porque en estas converge otra variable: lo transfronterizo.

Otro factor por considerar es la construcción de regímenes ambientales marinos y de una comunidad marina basada en un enfoque integral consolidado, puesto que ello resulta cuando "...los Estados que comparten un cuerpo de agua ecológico y socioeconómico común desarrollan instituciones comunes para su protección" (Chung, 2010; Hearns et al., 2014).

Un punto medular es cómo se delimita, para efectos jurídicos y políticos, el espacio del ecosistema. Porque según D. Langlet (2018) los regímenes legales de gobernanza marina incluyen conceptos como 'ecosistema', 'cuerpo de agua' y 'aguas transicionales' que suponen una naturaleza espacial.

La cuestión es que las confusiones pueden minar “...la efectividad del sistema regulatorio y perturbar el intercambio crucial de información entre ciencia y derecho" (Langlet, 2018). Sin olvidar, los factores e intereses políticos en juego en la zona fronteriza. Así a lo largo de la línea divisoria de los espacios marítimos/ marinos convergen lo natural, científico, jurídico, económico y político. Entonces la gestión de frontera desde el ecosistémica se debe reflejar la legislación nacional e internacional y el marco institucional marino y marítimo, reconociendo la interacción entre los sistemas natural y regulatorio, lo cual significa que la gestión pública y la gobernanza deben reconocer el cambio a través del tiempo y no solo las condiciones físicas y procesos, así como las variaciones en la tecnología, las técnicas, los conceptos científicos y los objetivos políticos; sin olvidar que los conceptos y constructos legales y políticos “...usados para describir, cuantificar y evaluar diferentes aspectos del ambiente marino también constituyen un marco conceptual que influencia las observaciones y evaluaciones" (Langlet, 2018).

No se puede obviar que los Estados ribereños no siempre tienen clara su responsabilidad con la administración del espacio marítimo/ marino y sus recursos naturales. Muchos gobiernos reivindican sus derechos marítimos sin consideración de sus habilidades para asumir las responsabilidades de la administración oceánica (Prescott \& Schofield, 2005).

Pero no se trata solo de la gestión del espacio marítimo y de la frontera, sino que en algunos casos se deben tener en cuenta otros factores. Por ejemplo, en el Pacífico centroamericano hay que reconocer lo que se denomina "Guardian Bank" (Prescott \& Schofield, 2005), junto con el llamado "domo térmico", que Costa Rica promueve como una "zona marina de importancia ecológica o biológica" (Jiménez, 2016).

Este tipo de factores agregan nuevos elementos a la gestión de las fronteras y la gobernanza marítima/marina. Entre los aspectos a considerar está la "densidad relacional" de la zona fronteriza, vinculada con la perspectiva que tienen los Estados sobre la interacción a lo largo de la línea divisoria, pues hay que tener en cuenta que en ese espacio se procura minimizar el riesgo de la interacción y “... se explora la creación de escenarios de cooperación $-\mathrm{y}$ por tanto de suma positiva-, y se diseñan los mecanismos e instrumentos de regulación que disminuyen la incertidumbre y el conflicto y acrecientan la confianza mutua" (Oddone, 2014).

Pero también se pueden generar las condiciones opuestas. Esto último dificulta la gestión fronteriza y la cooperación para resolver los problemas de diversa naturaleza que ocurren en el límite. De ahí la necesidad de tener en cuenta si los Estados colindantes tienen programas y políticas específicas, atendiendo, por ejemplo, la planificación espacial marina y marítima, la gestión de zonas costeras, de un manejo integrado del océano y áreas marinas protegidas. Esto es importante, porque no se puede pensar en el mar como un espacio homogéneo, en la medida que hay áreas de alta 
biodiversidad y productividad o criaderos, al igual la existencia de recursos no vivos; pero también de las actividades humanas que tienen lugar en los ecosistemas. Es decir, no se puede separar lo marino y lo marítimo, así como la relación espacio-temporal, en términos de la gobernanza. Esto debe basarse en un plan de gestión espacial, entendido como “...un documento integral y estratégico que proporciona un marco y una orientación para las decisiones de gestión espacial marina" (Comisión Oceanográfica Intergubernamental, 2013).

Sin embargo, en el caso de asuntos marinos no puede dejarse de lado el contexto regional, porque en algunos casos es necesario pensar en términos de los grandes ecosistemas marinos y de los acuerdos internacionales para la protección de los recursos, lo que hace necesario pensar en términos de gobernanza regional y un enfoque integrado (Chung, 2010). Un ejemplo de este tipo de enfoque es Asia Noroccidental y el Plan de Acción del Pacífico Noroeste (Chung, 2010). Pero también está la experiencia del Corredor Marino del Pacífico Este Tropical (Murillo, 2018). Esto demuestra que es posible implementar un enfoque de gestión ecosistémica en una región, aunque esta se caracterice por su heterogeneidad, producto de condiciones socioeconómicas, instituciones políticas, desarrollo histórico y herencia cultural (Kern \& Söderström, 2018), para lograr una buena gobernanza marina y marítima.

Tal planificación adquiere mayor relevancia cuando se trata de zonas fronterizas, porque los gobiernos deben coordinar acciones, teniendo en cuenta que el espacio marítimo está dividido, pero que el espacio natural marino está integrado. Sin olvidar que en la dimensión marítima operan distintas jurisdicciones y hay un registro histórico de las experiencias positivas y negativas. Esto se puede observar en el caso de Costa Rica y Nicaragua.

\section{Frontera Costa Rica - Nicaragua en el océano Pacífico}

La experiencia centroamericana en materia de fronteras terrestres es compleja por las rivalidades entre los Estados. Tal complejidad se extendió al espacio marítimo, en el cual no se han acordado la mayoría de las fronteras, excepto las de Costa Rica y Panamá. Mientras que las de Nicaragua, en ambas costas, fueron resueltas por sentencias de la Corte Internacional de Justicia. Y persisten las diferencias en el Golfo de Fonseca, a pesar de algunos tratados limítrofes y juicios ante la Corte de Justicia Centroamericana y la Internacional de Justicia (CIJ) (Montiel, 2002).

La mayoría de los diferendos están enquistados en la herencia colonial y conforme se han descubierto recursos naturales en la ZEE y la plataforma continental del Caribe y el Pacífico, las tensiones limítrofes aumentan (de Faramiñán, 2018). Esto pasa por las dificultades que tienen los países centroamericanos para lograr la integridad territorial, como ocurre en Nicaragua (de Faramiñán, 2018). Lo cual se extiende a las costas, por las características geográficas y naturales de estas, vulnerables a los cambios geomorfológicos y los efectos de los desastres naturales.

La historia de la frontera terrestre entre estos dos países es de larga data, y requirió de varias iniciativas en el siglo XIX para lograr un tratado limítrofe. Pero la línea divisoria acordada en 1858 no puso fin a las diferencias, requiriendo la intervención de árbitros (laudos Cleveland y Alexander). Inicialmente por el asunto del canal interoceánico (Murillo, 1986), luego por cuestiones de navegación del río San Juan y en el caso de los espacios marítimos por el interés de Nicaragua sobre los hidrocarburos existentes en el subsuelo cercano a la frontera con Costa Rica.

En el trabajo de Murillo (1986) se evidencia que la cuestión limítrofe no resultó del todo satisfactoria para las partes, sobre todo para Managua, por lo que las controversias persistieron para allá de los laudos. Por consiguiente, esta cuestión es conocida por la CIJ en varios casos $(2010,2011,2014$ y 2017) que fueron resueltos mediante sentencias de 2015 y 2018.

Existen factores que complejizan las relaciones bilaterales y particularmente la gestión de los espacios marinos/marítimos, porque si 
bien existen pocas bahías multiestatales -que pueden tener aguas comunes o divididas- en el mundo, estos dos países tienen dos: bahía Salinas y San Juan del Norte. En estas dos bahías lo que existe es un condominio, porque Costa Rica y Nicaragua comparten algunos derechos (Samuels, 2008). En ambos casos son aguas interiores.

La CIJ conoció el asunto de la delimitación marítima en el Pacífico, utilizando los criterios y métodos que ha implementado en las últimas sentencias, identificando las características de las costas de ambos países y en ellas los puntos base relevantes, a partir de los cuales se trazará la equidistancia provisional. La jurisprudencia de la corte establece que primero se traza la línea media y luego se ajusta a partir de las circunstancias especiales. Tal situación generó, una vez más diferendos entre las dos partes. El primer paso fue delimitar el mar territorial y luego la ZEE.

En la sentencia de febrero de 2018 se estableció la frontera marítima en el Pacífico a partir del punto medio de la línea que cierra bahía Salinas. Esto fue calificado por la prensa nicaragüense como un fallo en contra del país, aunque entidades gubernamentales lo calificaron de un éxito. Mientras que el gobierno costarricense, en un comunicado de prensa de la presidencia lo declaró un "histórico fallo", porque acogió los argumentos de Costa Rica, permitiendo el acceso garantizado al domo térmico y evitando que Nicaragua se hiciera de espacios marítimos en los que pretendía explorar y explotar petróleo y gas (Gobierno de Costa Rica, 2018).

En criterio de N. Boeglin (2018) el punto medular es que Costa Rica y Nicaragua carecen de un tratado de límites marítimos que defina el límite, pero sobre todo establezca los mecanismos de coordinación para la gestión de la frontera.

Al presentar el nuevo mapa oficial en abril de 2018, el entonces canciller costarricense Manuel González manifestó que el país “... no pretende quitarle nada a nadie, simplemente reclamar lo que es justo, según el derecho internacional y los análisis técnicos" (citado
Cambronero, 2018). Agregó, en esa oportunidad, que el fallo "...da los límites exactos para los dos países en el Pacífico como el Caribe, y podemos tener mayor certeza y mayor seguridad. En sentido literal, estamos entregando una Costa Rica más grande, más extensa”.

Los dos gobiernos establecieron en la década de 1990 la Comisión Binacional Costa Rica-Nicaragua. En ese foro se celebraron cinco encuentros; sin embargo, el tema de frontera marítima y asuntos marinos no ocupó un lugar prioritario. Luego se estableció el Programa de desarrollo fronterizo Costa RicaNicaragua 2007-2010, en el cual también estuvo ausente el tema de la frontera marítima.

\section{Consideraciones finales}

Costa Rica y Nicaragua carecen de marcos de referencia para la gestión y gobernanza del espacio marino/marítimo fronterizo. Esto debilita la coordinación de acciones a partir de un enfoque ecosistémico integral para la protección de los recursos naturales, sobre todo vivos. La carencia de esto dificulta la gestión de la frontera, tanto en la zona costera como en el mar territorial, que tiene una actividad pesquera importante. Pero también en la ZEE, en donde opera la flota atunera y las actividades ilícitas.

Desde la perspectiva de la investigación científica, la falta de cooperación bilateral incide en el desarrollo de estudios marinos de carácter holístico. Mientras, queda pendiente de analizar la cuestión de las aguas interiores en condominio jurídico, pero no real, por las dificultades de coordinación gubernamental.

Si bien en el caso de la línea limítrofe terrestre de estos dos países también presenta problemas de coordinación, para operar como frontera. De la observación de la experiencia entre lo terrestre y lo marino, se concluye que la gestión pública de fronteras marítimas no puede ser la misma del límite terrestre, porque el espacio natural y geográfico y la construcción del territorio y la territorialidad tiene otros criterios; y tampoco se puede obviar lo relativo a gobernanza, por la relación que existe entre 
gestión y gobernanza. La práctica de Costa Rica y Nicaragua es un ejemplo de la necesidad de coordinar y cooperar en fronteras marítimas, y con mayor razón un tratado de límites que establezca los compromisos de las partes.

Es necesario revisar las políticas de ambos países sobre los mares y la planificación marina, para tener clara la gestión de fronteras marítimas; así como de las iniciativas académicas y privadas que involucren a ambos países. Por eso este trabajo es una primera aproximación a la temática.

Declaración de ética: el autor declara que está de acuerdo con esta publicación; que no existe conflicto de interés de ningún tipo; y que ha cumplido con todos los requisitos y procedimientos éticos y legales pertinentes. Todas las fuentes de financiamiento se detallan plena y claramente en la sección de agradecimientos. El respectivo documento legal firmado se encuentra en los archivos de la revista.

\section{RESUMEN}

Introducción: La gestión pública ha adquirido nuevas características, producto de cambio en la forma en que se aborda la gobernanza. Conforme las áreas temáticas, niveles y escalas de esa gestión se ha expandido, la administración pública se tornado más compleja, requiriendo nuevos enfoques. Si bien el derecho del mar y la tesis de que la tierra domina el mar, no son nuevas, lo cierto es que fue con la Convención de Naciones Unidas sobre el Derecho del Mar, de 1982, que se formaliza e institucionalizan los espacios bajo jurisdicción del Estado ribereño. Ello ha generado nuevo desafíos y retos para los gobiernos, pues no solo se extiende la soberanía territorial sobre una extensa porción del mar, sino que se asumen responsabilidades en nombre de la comunidad internacional.

Objetivo: Como se trata de una cuestión que vincula la gestión pública con la normativa del derecho del mar en materia de delimitación y administración de fronteras; por lo que en esta revisión se busca plantear una aproximación a un asunto sobre el cual se ha explorado poco.

Métodos: Se realizó una revisión de la literatura sobre gestión pública de fronteras considerando el caso de las fronteras marítimas, identificando jurisprudencia internacional y acciones implementadas por Costa Rica.

Resultados: En la porción terrestre la gestión de fronteras muestra un desarrollo importante; no así en los espacios marítimos. La jurisdicción es diferente en cada uno de esos espacios, por lo que la gestión pública debe tener en cuenta esas particularidades. Pero, las cosas se complican cuando se trata del espacio fronterizo, porque en este converge lo marino y lo marítimo, pero también diversos intereses, por la naturaleza del espacio marino. Por consiguiente, es necesario reconocer que la gestión de fronteras marítimas no es tan sencilla como trasladar lo que se hace en tierra al mar. Cuando el límite fue definido por un tratado bilateral, existen disposiciones particulares que facilitan la coordinación y cooperación transfronteriza; por eso se utilizó como referencia el caso Costa Rica y Nicaragua en el océano Pacífico, pues en este caso la línea divisoria la estableció la Corte Internacional de Justicia, lo que provoca un trazado que no necesariamente es satisfactorio para los actores involucrados.

Conclusiones: las diferencias en la gestión de fronteras entre lo terrestre y lo marítimo, sumado a la forma en que se definió el límite entre los dos países, ha dificultado la implementación de la gestión pública por parte de Costa Rica, que es la experiencia más conocida en la región sobre políticas y gestión marina-marítima.

Palabras clave: Derecho del Mar; fronteras marítimas; gestión pública; Costa Rica; Nicaragua; Océano Pacífico.

\section{REFERENCIAS}

Acikgonul, Y. E. (2016). Reflections on the Principle of Non-Cut Off: A Growing Concept in Maritime Boundary Delimitation Law. Ocean Development \& International Law, 47(1), 52-71. https://doi.org/10.1 080/00908320.2016.1124485

Aguilar, L. (2010a). El futuro de la gestión pública y la gobernanza después de la crisis. Frontera Norte, 22(43), 187-213.

Aguilar, L. (2010b). Gobernanza: el nuevo proceso de gobernar. Ciudad de México, México: Fundación Frieridch Naumann para la Libertad.

Aguilar, L. (2016). Gobernanza y gestión pública. Ciudad, México: Fondo de Cultura Económica.

Baldwin, K., Mahon, R., \& McConney, P. (2013). Participatory GIS for strengthening transboundary marine governance in SIDS. Natural Resources Forum, 37, 257-268. https://doi.org/10.1111/1477-8947.12029

Boeglin, N. (2018, 6 de febrero). Costa Rica - Nicaragua: La delimitación marítima decidida por la Corte Internacional de Justicia. América Latina en movimiento. https://www.alainet.org/es/articulo/190870

Cadena, W. R., \& Devia, C. A. (2012). Colombia y la territorialización de sus mares. Conflictos limítrofes y la Convención de Derecho del Mar. Revista Prolegómenos. Derechos y Valores, 15(30), 199-223. https://doi. org/10.18359/prole.2365 
Cambronero, N. (2018, 23 de abril). Costa Rica presenta su nuevo mapa con $36.000 \mathrm{~km}^{2}$ maritimos ganados a Nicaragua en La Haya. La Nación. https://www.nacion.com/el-pais/politica/costarica-presenta-su-nuevo-mapa-con-36000-km/ KGGGDGR3LNC5BAIFRODJBAGV24/story/

Caron, D. (2009). Climate Change, Sea Level Rise and the Coming Uncertainty in Oceanic Boundaries: A Proposal to Avoid Conflict. In S. Hong, \& J. Van Dyke (Eds.), Maritime Boundary Disputes, Settlement Processes, and the Law of the Sea (pp. 1-17). Leiden, Netherlands: Martinus Nijhoff Publishers.

Celata, F., Coletti, R., \& Sanna, V. S. (2013). La cooperación transfronteriza en la región del del Trifinio y la difusión de modelos europeos de gobernanza de las fronteras en América Latina. Si Somos Americanos. Revista de Estudios Transfronterizos, $\operatorname{XIII}(2)$, 165-189. https://doi.org/10.4067/ S0719-09482013000200008

Chung, S. (2010). Strengthening regional governance to protect the marine environment in Northeast Asia: From a fragmented to an integrated approach. Marine Policy, 34, 549-556. https://doi.org/10.1016/j. marpol.2009.10.011

Comisión Oceanográfica Intergubernamental. (2013). Planificación Espacial Marina. Una guía paso a paso hacia la Gestión Económica. http://mspes. ioc-unesco.org/wp-content/uploads/2016/05/Planificacion-Espacial-Marina.pdf

De Faramiñán, J. (2018). Las diferencias fronterizas en Centroamérica. Anuario Español de Derecho Internacional, 34, 265-300. https://doi. org/10.15581/010.34.265-300

División de Asuntos Oceánicos y del Derecho del Mar. (2001). Manual de delimitación de fronteras maritimas. Nueva York: Naciones Unidas.

Elizalde, A. (2003). Planificación estratégica territorial y políticas públicas para el desarrollo local. Santiago, Chile: ILPES-CEPAL

Evans, M. (2016). Maritime Boundary Delimitation. In D. Rothwell, A. Oude-Elferink, K. Scott, \& T. Stephens (Eds.), The Oxford Handbook of the Law of the Sea (pp. 254-279). Oxford, UK: Oxford University Press. https://doi.org/10.1093/ law/9780198715481.003.0012

Flores, J. R. (2014). Observación y planificación estratégica territorial. San José, Costa Rica: ConectaDelFundación Demuca

Global Water Partnership Centroamérica. (2019). Politica Pública de las aguas transfronterizas en la región centroamericana. https://www.gwp.org/es/GWP-Centroamerica/CONOCIMIENTO/publicaciones-recientes/ politica-publica-de-las-aguas-transfronterizas/
Gobierno de Costa Rica. (2018, 2 de febrero). Histórico fallo de la Corte Internacional de Justicia acoge argumentos de Costa Rica en la delimitación maritima con Nicaragua. https://www.icesi.edu.co/revistas/ index.php/estudios_gerenciales/article/view/ 1114/ pdf

Gómez-Robledo, A. (2016). Apuntes sobre delimitación en derecho internacional del mar. Anuario Mexicano de Derecho Internacional, XVI, 255-300. https://doi. org/10.22201/iij.24487872e.2016.16.527

Haque, E., \& Hassan, D. (2014a). Maritime boundary delimitation: A normative standard? Part I: Historical development of the legal regime. Environmental Policy and Law, 44(5), 433-450.

Haque, E., \& Hassan, D. (2014b). A normative standard? Part II: Is it still insufficiently addressed? Environmental Policy and Law, 44(6), 517-530.

Hearns, G., Henshaw, T., \& Paisley, P. (2014). Getting what you need: Designing institutional architecture for effective governance of international waters. Environmental Development, 11, 98-111. https://doi. org/10.1016/j.envdev.2014.04.005

Henshaw, T. (2018). What Works in Water and Ocean Governance: Impact Stories from the UNDP Water and Ocean Governance Programme. UNDP. https:// fundingtheocean.org/reports/what-works-in-waterand-ocean-governance-impact-stories-from-theundp-water-and-ocean-governance-programme/

Jiménez, J. (2016). El domo térmico de Costa Rica: Un oasis de productividad frente a las costas del Pacifico Centroamericano. San José, Costa Rica: MarViva.

Kern, K., \& Söderström, S. (2018). The ecosystem approach to management in the Baltic Sea Region: Analyzing regional environmental governance form a spatial perspective. Marine Policy, 98, 271-277. https://doi.org/10.1016/j.marpol.2018.09.023

Lacleta, J. M. (2003). Fronteras en el mar. Politica, derecho y equidad en la delimitación de los espacios marinos. WP23./2003. Real Instituto Elcano. http:// www.realinstitutoelcano.org/wps/portal/rielcano es/ contenido?WCM GLOBAL CONTEXT=/elcano/ elcano es/zonas es/dt23-2003

Langlet, D. (2018). Scale, space and delimitation in marine legal governance. Perspectives from the Baltic Sea. Marine Policy, 98, 278-285. https://doi. org/10.1016/j.marpol.2018.09.027

Lemons, M., \& Agrawal, A. (2006). Environmental governance. Annual Review of Environmental and Resources, 31, 297-325. https://doi.org/10.1146/annurev. energy.31.042605.135621

Lizama, N. (2017). La gobernanza ambiental transfronteriza como perspectiva para estudiar la conflictividad socioambiental en torno a la minería en zona 
de frontera. Revista Iberoamericana de Viticultura, Agroindustria y Ruralidad, 4(11), 52-73.

Martínez, L. (2012). Apuntes para pensar el territorio desde una dimensión social. Ciencias Sociales Unisonos, 48(1), 12-18. https://doi.org/10.4013/ csu.2012.48.1.02

Montiel, A. (2002). El Golfo de Fonseca. Encuentro, 62, 64-84. https://doi.org/10.5377/encuentro.v0i62.4167

Moyado, F. (2011). Gobernanza y calidad de la gestión pública. Estudios Gerenciales, 27(120), 205-223.

Murillo, C. (2017). Costa Rica y el derecho del mar, $2^{\mathrm{a}}$ ed. San José, Costa Rica: Editorial UNED.

Murillo, C. (2018). Declaración de San José sobre el Corredor Marino del Pacífico Este Tropical. Alcance naturaleza jurídica y relevancia para la gestión de la biodiversidad marina y la gobernanza oceánica. In M. García (Ed.), Tratado de derecho de aguas: Tomo II: perspectiva internacional del derecho de aguas para el siglo XXI (pp. 349-381). Bogotá, Colombia: Universidad Externado de Colombia.

Murillo, H. (1986). La controversia de límites entre Costa Rica y Nicaragua. El Laudo Cleveland y los derechos canaleros 1821-1903. Anuario de Estudios Centroamericanos, 12(2), 45-58.

Oddone, N. (2014). Cooperación transfronteriza en América Latina: Una aproximación teórica al escenario centroamericano desde la experiencia del Proyecto Fronteras Abiertas. Oikos, 13(2), 129-144.

Organización Internacional para la Migraciones. (2012) Módulo I: Gestión de fronteras. Gestión fronteriza integral para la subregión andina. Lima, Perú: Organización Internacional para la Migraciones. https:// peru.iom.int/sites/peru/files/Documentos/Modulo1.pdf
Orihuela, E. (2017). La delimitación de espacios maritimos entre Estados y la jurisprudencia internacional. Cursos de derecho internacional y relaciones internacionales de Vitoria-Gasteiz, No 1, 487-574. https://www. ehu.eus/es/web/cursosderechointernacionalvitoria/-/ la-delimitacion-de-espacios-marinos-entre-estadosy-la-jurisprudencia-internacional

Prescott, V., \& Schofield, C. (2005). The Maritime Political Boundaries of the World. Leiden, Netherlands: Martinus Nijhoff Publishers.

Samuels, J. (2008). Condominium Arrangements in International Practice: Reviving an Abandoned Concept of Boundary Dispute Resolution. Michigan Journal of International Law, 29(4), 727-776.

Sassen, S. (2013). Territorio, autoridad y derechos. De los ensamblajes medievales a los ensamblajes globales. Madrid, España: Katz Editores.

Sobrino, J. M., \& Oanta, G. A. (2010). Control y vigilancia de las fronteras en los diferentes espacios marítimos. Anuario da Facultade de Dereito da Universidade da Coruña, 14, 759-788. https://core.ac.uk/download/ pdf/61902936.pdf

Spíndola, O. (2016). Espacio, territorio y territorialidad: una aproximación teórica a la frontera. Revista Mexicana de Ciencias Políticas y Sociales, 228, 27-55. https://doi.org/10.1016/S0185-1918(16)30039-3

Talaie, F. (1999). The Issue of the Delimitation of MultiState Bays in the International Law of the Sea. University of Tasmania Law Review, 18(1), 22-64.

Vargas, G. (2012). Espacio y territorio en el análisis geográfico. Revista Reflexiones, 91(1), 313-326. 